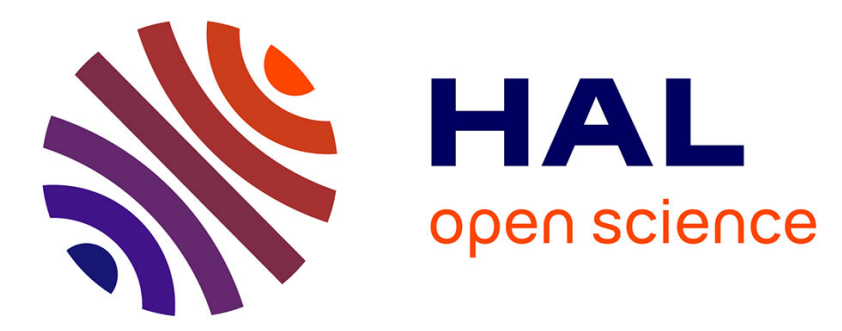

\title{
Entre discordance et ajustement inter-énonciatifs
}

Agnès Celle

\section{To cite this version:}

Agnès Celle. Entre discordance et ajustement inter-énonciatifs. Colloque international Représentations du Sens Linguistique, III, Nov 2005, Bruxelles, Belgique. pp.133-147, 10.3917/dbu.evrar.2009.01.0133 . hal-01227266

\section{HAL Id: hal-01227266 \\ https://hal-univ-paris.archives-ouvertes.fr/hal-01227266}

Submitted on 19 Feb 2021

HAL is a multi-disciplinary open access archive for the deposit and dissemination of scientific research documents, whether they are published or not. The documents may come from teaching and research institutions in France or abroad, or from public or private research centers.
L'archive ouverte pluridisciplinaire $\mathbf{H A L}$, est destinée au dépôt et à la diffusion de documents scientifiques de niveau recherche, publiés ou non, émanant des établissements d'enseignement et de recherche français ou étrangers, des laboratoires publics ou privés. 


\title{
ENTRE DISCORDANCE ET AJUSTEMENT INTER- ENONCIATIFS
}

\author{
Agnès CELLE \\ Université Paris 7 Denis-Diderot
}

Maintenant en français, now en anglais, et nun en allemand ont en commun la capacité de faire référence au moment de l'énonciation dans leur emploi temporel, et de jouer un rôle discursif en introduisant une nouvelle étape dans l'argumentation, voire en reformulant un dire. C'est cet emploi de marqueur discursif ${ }^{1}$ qui va nous intéresser plus particulièrement dans cette étude. Si ces trois marqueurs présentent une grande proximité en termes temporels - ils renvoient tous à $\mathrm{T}_{0}$ - leurs emplois discursifs en revanche sont divergents et notre objet ici est de mettre au jour la façon dont ils construisent le sens. Dans l'approche énonciative que nous adoptons, c'est en termes d'altérité que s'analyse la relation entre les domaines notionnels mis rapport par ces trois marqueurs. Mais au-delà de la nature de l'altérité mise en jeu, il faudra également se demander comment ces marqueurs construisent la relation coénonciative.

\section{La distinction entre adverbe temporel et marqueur discursif}

La plupart des travaux sur des marqueurs polysémiques distinguent l'adverbe temporel de la particule modale, parfois même de la particule connective ou focale. La catégorisation est malaisée. En tout cas, il semble généralement admis que la valeur temporelle est exprimée par un adverbe, tandis que la dénomination de l'emploi discursif est variable. Dans un article récent, Paillard soutient au contraire que tous les emplois de déjà, y compris aspectuotemporels, relèvent du marqueur discursif. Même si la distinction entre adverbe et marqueur discursif reste à affiner, le test de la négation est révélateur et, dans le cas des trois marqueurs qui nous occupent ici, il permet d'opérer une première différence : en effet, maintenant et now peuvent être niés, alors que nun ne peut être nié en tant que tel :

\footnotetext{
Viele kennen das Jetzt nicht Syndrom. Das Jetzt nicht Syndrom tritt immer dann auf, wenn unbequeme Dinge erledigt werden müssen. $<$ http://www.bildungslexikon.info/jetztnicht.html $>$

[Beaucoup connaissent le syndrome du «pas maintenant». Le syndrome du «pas maintenant » se manifeste toujours lorsqu'il faut faire des choses peu agréables.] $]^{2}$
}

Il s'agit ici simplement de nier l'adverbe temporel jetzt. Le Nun nicht Syndrom n'aurait aucun sens, car nun n'est pas seulement repère temporel. De la même façon, dans la traduction de now nié dans l'exemple suivant, nun ne peut apparaître :

$$
\text { Yes ... Well, I can't talk now... I can't talk now, old sport... (F. Scott Fitzgerald, The Great }
$$
Gatsby, p. 91)

\footnotetext{
Nous exprimons notre gratitude à $\mathrm{C}$. Blanche-Benveniste qui a bien voulu nous transmettre un concordancier des emplois de maintenant tiré du corpus oral CORPAIX. La comparaison de maintenant et now dans leur emploi discursif est largement reprise de Celle (2004).

2 Les traductions entre crochets sont les nôtres. Les autres sont des traductions publiées.
} 
Ja, aber ich kann jetzt hier nicht sprechen... Ich kann jetzt nicht sprechen, alter Junge... (W. Schürenberg, L. Blanvalet, p. 139)

Il ne s'agit pas d'une incompatibilité entre le marqueur et la négation, mais le sens produit ne correspond pas à la simple négation du marqueur. Dans la traduction de l'exemple (2), nun nié voudrait dire non pas « je ne peux pas parler maintenant», mais « je ne peux plus parler maintenant ». C'est-à-dire que la négation mettrait le procès en relation avec autre chose : l'impossibilité présente de parler résulterait d'un fait antérieur - langue coupée ou interdiction de parler par exemple. La substitution avec nun n'est pas impossible dans l'exemple suivant, mais on observe la même différence de sens :

Wie genau, weiß er noch nicht, aber loslassen kann er jetzt nicht. (Der Spiegel). [Comment exactement, il ne sait pas encore, mais il ne peut pas lâcher prise maintenant.]

Avec jetzt nicht, il n'est pas possible de lâcher prise maintenant, ce qui laisse ouverte la possibilité de lâcher prise à un autre moment. Avec nun nicht, cette possibilité existait, mais n'existe plus maintenant. On peut à présent mieux comprendre pourquoi en (1) le Nun nicht Syndrom n'a guère de sens : il manque l'altérité indispensable au fonctionnement de nun, et c'est la relation à celle-ci qui est à l'origine de la différence de sens entre jetzt et nun que l'on perçoit nettement à la négation. Si l'on représente par $\mathrm{p}$ nun q l'enchaînement discursif, nun q implique que $\mathrm{p}$ n'est pas stabilisé. C'est le point de vue nun q qui va le stabiliser. Nun ne peut construire à la négation «q n'est pas le cas maintenant» car cette séquence ne s'inscrit pas dans une relation à $\mathrm{p}$ que nun pourrait stabiliser. La lecture « $\mathrm{q}$ n'est plus le cas maintenant » suppose au contraire l'altérité.

Si l'on ne peut reconstruire une altérité en fonction contexte avant, la substitution avec nun devient problématique et il faut ajouter mehr à la négation (ne plus) pour que l'interprétation en termes de discontinuité redevienne possible, autorisant ainsi la mise en place d'un autre point de vue :

Später, bin jetzt nicht aufgelegt zu einer Probe... habe andere Sachen im Kopf. Geh jetzt! Komm! (P. Süskind, das Parfum, p. 110) [Plus tard; je ne suis pas d'humeur à essayer maintenant... J'ai d'autres choses en tête. Pars maintenant! Allez !]

?Später, bin nun nicht aufgelegt zu einer Probe... habe andere Sachen im Kopf.

Später, bin nun nicht mehr aufgelegt zu einer Probe... habe andere Sachen im Kopf.

La négation est un indice parmi d'autres du statut différent de nun par rapport à jetzt, maintenant et now. En dehors des énoncés négatifs, on retrouve cette différence dans le rapport à $\mathrm{p}$ et dans le point de vue que sous-tend $\mathrm{q}$ :

Die Welt machte mich zu einer Hure, nun mache ich sie zu einem Bordell. (F. Dürrenmatt,

Besuch der alten Dame) [Le monde a fait de moi une putain, et maintenant je fais du monde un bordel.]

Nach einem dreimonatigen Auslieferungsstopp wird Daimler-Benz am 9. Februar seine Händler mit der technisch aufgerüsteten A-Klasse beliefern. [...] Die A-Klasse wird nun mit einer neuen Fahrwerkabstimmung, einer deutlichen Absenkung der Karosserie, mit einer neuen Räder-Reifen-Kombination und vor allem mit dem elektronischen Fahrdynamiksystem ESP auf den Markt kommen. (Stuttgarter Zeitung 17.1.98)

[Après une suspension des livraisons de trois mois, Daimler-Benz va approvisionner ses concessionnaires, le 9 février, en classes A plus performantes. [...] La classe A va maintenant / à présent revenir sur le marché avec un nouveau réglage de direction, un net nivellement de la carosserie, avec un nouveau jeu de pneumatiques, et avant tout avec le système électronique de conduite dynamique E.S.P.] 
Nun marque une nouvelle étape qui s'inscrit dans une logique de conformité. En (5), nun signifie que du point de vue du locuteur, q n'est que le logique et juste retour des choses, étant donné p. En (6), nun insère q dans un processus, celui de la commercialisation de la voiture en question, compte tenu des modifications techniques qui lui ont été apportées et qui sont mentionnées en p. Jetzt, au contraire, n'entretient aucun lien avec ce qui précède :

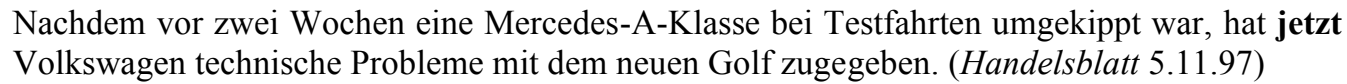

[Après qu'une classe A de Mercedes se fut retournée lors de parcours d'essais il y a deux semaines, c'est maintenant au tour de Volkswagen de reconnaître des problèmes techniques avec la nouvelle Golf.]

Les problèmes techniques que rencontre Volkswagen sont totalement indépendants de ceux de Mercedes et leur survenue en $\mathrm{T}_{0}$ est contingente. Jetzt a uniquement pour rôle d'ancrer le procès de la proposition en $\mathrm{T}_{0}$ et aucun point de vue n'est associé à q. Jetzt est un adverbe temporel et n'a pas d'emploi discursif. Nun, au contraire, est un marqueur discursif, ce qui explique que même dans ses emplois dits temporels, il ne soit pas équivalent à jetzt : il introduit toujours en $q$ un point de vue relatif à la séquence $p$.

Now et maintenant peuvent être adverbes temporels, comme jetzt, mais aussi marqueurs discursifs $^{3}$, comme nun, et c'est à l'emploi discursif de ces trois marqueurs et à leur comparaison que nous allons nous intéresser à présent.

\section{L'emploi discursif de maintenant, now et nun}

\subsection{Maintenant et now}

\subsubsection{L'articulation des valeurs temporelle et discursive}

Doit-on considérer que, dans le cas de maintenant et de now, les emplois temporels et discursifs relèvent de deux marqueurs différents, ou bien peut-on au contraire rassembler ces deux emplois autour de la valeur centrale de chaque marqueur? Nef (1980:163) est partisan de la première approche. Il distingue maintenant 1 , « sémantique-temporel », de maintenant 2 , «pragmatique-discursif » dont la fonction est de « connecter pragmatiquement des actes de langage ».

Du côté de l'anglais, la problématique est la même. Aijmer (2002: 57-63) distingue l'adverbe temporel de la particule discursive (discourse particle) dont le rôle est d'indiquer « a boundary or a 'frame' between two discourse units » (p. 53). Elle reconnaît néanmoins, comme Schiffrin (1987: 231), qu'il existe une zone floue « where the discourse structure of temporal comparisons neutralizes the distinction between now as a time adverb and now as a

\footnotetext{
La question de savoir s'il s'agit d'un marqueur discursif ou d'un adverbe n'est pas simplement une question d'ordre terminologique. Comme le souligne Paillard, c'est un enjeu sémantique. « un adverbe relève [...] d'une sémantique intrapropositionnelle : son champ est la proposition où il intervient pour spécifier tel ou tel composant de la relation prédicative. Inversement, un MD [marqueur discursif] relève d'une autre sémantique : il définit le statut discursif de la séquence correspondant à sa portée. »
} 
discourse marker» (Schiffrin 1987: 231). En effet, si l'on peut identifier aisément le marqueur discursif lorsque le temps verbal renvoie au passé comme dans l'exemple suivant,

\author{
She had a cousin, woman cousin. Yes. Now, this woman found the letters and took them to $\mathrm{Mr}$ \\ Gosse, asked him his opinion of their value. (J. Barnes, Flaubert's Parrot, p. 43)
}

Elle avait une cousine. Oui. $\varnothing$ Cette femme trouva les lettres, les porta à M. Gosse en lui demandant ce qu'elles valaient... (J. Guiloineau, p. 51)

cette identification devient plus délicate lorsque now introduit une 'comparaison temporelle' (selon les termes de Schiffrin $1987: 231$ et Aijmer $2002: 60-61$ ) qui coïncide en $\mathrm{T}_{0}$ avec une nouvelle étape dans le discours (tournant dans la conversation, nouvel argument) :

$$
\begin{aligned}
& \text { er what is it?, what are they complaining about? Tax reductions, of that's nice, interest rates or } \\
& \text { something and those said oh they're too high over here, in America they're four per cent, now } \\
& \text { what they don't see is the interest rates are linked with the amount of peop the amount of } \\
& \text { people earn, the amount of people actually save (BNC Spoken) }
\end{aligned}
$$

De la même façon en français, les deux valeurs ne s'excluent pas forcément:

\footnotetext{
Votre affaire n'est pas mauvaise, dit Athos après avoir goûté le vin en connaisseur et indiqué d'un signe de tête qu'il le trouvait bon, et l'on pourra tirer de ce brave homme cinquante à soixante pistoles. Maintenant, reste à savoir si cinquante à soixante pistoles valent la peine de risquer quatre têtes. (A. Dumas, les Trois Mousquetaires)
}

Si l'on avait le marqueur en position intrapropositionnelle (what they don't see now en (9) et Reste à savoir maintenant en (10)), la valeur temporelle serait seule présente. Mais la position antéposée, voire détachée de ces marqueurs favorise l'émergence de la valeur discursive. Rien ne s'oppose pour autant à une lecture temporelle, et il est difficile de déterminer dans quelle mesure les deux valeurs coexistent. On disposerait de plus de critères pour cela à partir de données orales enregistrées qu'à partir de textes écrits. En ce qui concerne l'anglais, now discursif, à la différence de now temporel, n'est pas accentué (cf. Halliday \& Hasan 1976 : 268, Aijmer 2002 : 66-83). En français, il semble que maintenant discursif ait une intonation non finale ${ }^{4}$, à la différence de maintenant temporel. La proximité des deux valeurs, en tout cas à l'écrit, rend ici possible aussi bien une interprétation adverbiale qu'une interprétation discursive, ce qui nous incite à penser que l'on doit chercher à représenter le sens de maintenant et de now en prévoyant cette variation, et sans opposer le marqueur temporel au marqueur discursif. Comme le suggère d'ailleurs Nef (1980:162), c'est par le biais de la deixis que l'intégration des deux descriptions peut se faire. Dans tous les cas en effet, maintenant et now réfèrent au moment de l'énonciation. Ce qui peut varier, c'est ce avec quoi $\mathrm{T}_{0}$ est mis en rapport. Soit maintenant / now fournit le repère temporel du procès à l'intérieur de q, auquel cas le marqueur joue un rôle adverbial dans cette proposition ; par rapport à $\mathrm{p}$, cela implique une discontinuité. Soit maintenant / now articule la séquence discursive par rapport à $\mathrm{T}_{0}$; par rapport à $\mathrm{p}$, cette mise en relation implique aussi une discontinuité, mais celle-ci ne vaut pas en termes aspectuo-temporels : il ne s'agit pas dans ce cas de différencier des intervalles, mais des points de vue concernant un état de choses. Les marqueurs discursifs maintenant et now vont affecter q d'un point de vue et manifester la différenciation entre deux séquences articulées autour de deux partenaires énonciatifs. C'est là que dans une perspective comparative, il y a variation. 


\subsubsection{Comparaison des marqueurs discursifs maintenant et now}

Les marqueurs discursifs now et maintenant apparaissent en position initiale ${ }^{5}$, maintenant étant plus souvent en position détachée. Ils introduisent un point de vue en $\mathrm{T}_{0}$ qui différencie la séquence de la précédente. Ce rapport à l'altérité leur est commun, et leur correspondance en traduction en est un reflet. La référence à une altérité apparaît tout particulièrement dans les deux langues lorsque le marqueur est suivi d'une hypothétique, d'une interrogative directe ou indirecte comme en (10) ou dans les exemples suivants :

Son, I'm busy, now if there was anything else you wanted... (C. McCarthy, The Orchard Keeper, p. 233)

Mon petit, j'ai du travail, maintenant si vous avez encore besoin de quelque chose... (Hirsch, F. \& Schaeffer P., p. 273)

Well, whatever you have, that's it! You've got to learn to live with it. Now! The question is 'How are you going to live with it?' (Brown Corpus)

On continuera de soutenir les revues. Maintenant, quelle va être la contribution de l'Université ? Nous ne savons pas encore. (conseil scientifique d'UFR, octobre 2005)

Dans ces exemples, la séquence $\mathrm{p}$ est prise en charge par l'énonciateur. Now / maintenant marque ensuite une bifurcation vers "autre chose ", et introduit un parcours marqué par une hypothétique, une interrogative, ou une incomplétude. En (11), l'énoncé inachevé est ensuite laissé en suspens, ce qui signale l'incapacité de l'énonciateur de sélectionner une valeur et une seule. Cette instabilité laisse au coénonciateur une possibilité de choix entre les deux valeurs suggérées. On constate qu'à chaque fois, now marque le passage d'un énoncé stabilisé à un énoncé non assertif où l'énonciateur ne peut sélectionner une valeur unique. Dans ces emplois, now et maintenant mettent en jeu les mêmes opérations. Ils indiquent un changement dans la prise en charge subjective de l'énoncé. Cette valeur peut se gloser ainsi : «p est le cas, now / maintenant q est une autre relation que je ne peux asserter ». L'altérité qualitative s'appuie ici sur une altérité intersubjective.

Toutefois, la construction de l'altérité avec maintenant peut aller plus loin. Suivi d'un énoncé incomplet ou d'une assertion, maintenant construit un nouveau domaine en réorientant le domaine précédent. Maintenant est le seul des trois marqueurs considérés à pouvoir effectuer cette opération de nature réflexive. oui oui en plus je suis pressé / maintenant quand même / / (CORPAIX)

Ça [la fusion des équipes] ne change rien. Maintenant, du point de vue administratif, ça va être compliqué à gérer. (conseil scientifique d'UFR, octobre 2005)

La question du jour : Les délinquants sexuels doivent-ils porter le bracelet électronique?

Alexis - 21 ans Etudiant

«Je pense que c'est assez intéressant pour permettre une réinsertion correcte et pour lutter contre les récidivistes. Maintenant, il est vrai que c'est un peu dur dans la mesure où porter un bracelet électronique c'est un manque total de liberté. » (Métro, Paris, 29-09 2005, n 803).

L2 c'est appréhendable par tout le monde L1 hum L3 + bon maintenant il y a des explications scientifiques qui sont compliquées (CORPAIX)

Maintenant discursif est toujours à l'initiale. Now discursif aussi en général, mais on peut également rencontrer well now, why now, say now, there now, et le redoublement du marqueur: now now. 
Dans les exemples (14) à (17), comme précédemment, p contient un énoncé stabilisé doté d'une orientation. Mais à la différence des exemples précédents, q est dotée d'une orientation contraire et maintenant fait de q le repère, ce qui déstabilise le premier point de vue. : p devient discutable. Maintenant q correspond à une altérité prise en charge par l'énonciateur. Cette différence par rapport à now devrait apparaître plus clairement si l'on compare (14) et (11), qui sont tous deux des énoncés incomplets. L'incomplétude va en effet s'interpréter de façon différente. En (11), le client parle depuis un moment avec l'employée et celle-ci s'impatiente. Les points de suspension sous-entendent « hurry up, because I don't have much time ». Now introduit un nouveau domaine dans lequel le coénonciateur est le point de référence. L'interlocuteur peut choisir de se presser ou de ne pas se presser. Mais ce nouveau domaine, tout en étant différent de celui posé en $\mathrm{p}, \mathrm{n}$ 'a pas une orientation contraire à celui-ci. L'employée s'attend simplement à ce que l'interlocuteur prenne en compte l'assertion préalable (I'm busy), au cas où il voudrait encore acheter quelque chose. Cela revient à lui conseiller de se dépêcher, et les deux relations n'ont rien de contradictoire.

L'exemple (14), contrairement à (11), ne permet pas cette interprétation. Maintenant y prend une valeur de concession rectificative qui peut se gloser par «Bien que je sois pressé, je peux le faire ». Maintenant introduit une orientation différente qui va à l'encontre de l'orientation de $\mathrm{p}$. On a là une mise en cause de $\mathrm{p}$ qui ne peut être effectuée par now en anglais. Contrairement à now, maintenant construit l'altérité en réidentifiant une propriété initiale par rapport au point de vue de l'énonciateur. Il y a donc discordance.

\subsection{Now et nun}

\subsubsection{Now et l'altérité du point de vue}

Contrairement à maintenant, now et nun ne peuvent avoir de fonction réflexive ${ }^{6}$. Examinons tout d'abord now :

\footnotetext{
'American society', she said, (...) 'not only sanctions gross and unfair relations among men, but it encourages them. Now, can that be denied ? No. Rivalry, competition, envy, jealousy, all that is malignant in human character is nourished by the system.' (P. Roth, Portnoy's Complaint, p. 237)

«La société américaine », dit-elle, (...) « non seulement sanctionne les relations barbares et injustes entre les hommes, mais encore elle les encourage. Voyons, peut-on le nier ? Non. La rivalité, la compétition, l'envie, la jalousie, tout ce qu'il y a de pernicieux dans le caractère de l'homme est alimenté par le système. (H. Robillot, p. 355)
}

En (18), l'énonciateur fait d'abord une évaluation dans un énoncé assertif. Ensuite, il reprend ce jugement dans une interrogation, où il cherche l'adhésion du coénonciateur. Now q ne modifie en rien l'orientation de la propriété notionnelle posée en p. Now q sollicite le coénonciateur pour qu'il confirme à son tour l'évaluation construite en p. Il n'y a pas altération de l'orientation de la première séquence, mais sollicitation d'un autre point de vue pour avaliser le précédent point de vue. La question rhétorique qui vient après (can that be denied ?) vise à élargir la validité de ce premier point de vue à tout un chacun, y compris le coénonciateur qui est ici sollicité fictivement.

\footnotetext{
$6 \quad$ Dans ce type d'énoncé, on aurait but et non now en anglais.
} 
Le rôle fondamental de now est de construire la relation coénonciative,ce qui explique d'ailleurs que ce marqueur, à la différence de maintenant, puisse apparaître sans qu'il y ait eu d'échange au préalable :

Frank Cornish received Miss Marple with cordiality and even deference. He set chairs for the two ladies, and said: 'Now what can I do for you, Miss Marple?' (A. Christie, 4.50 From Paddington, p. 14).

Say now, he said, you don't have any, uh - his eyes took a quick last inventory - any tire pumps, do ye ? (C. McCarthy, The Orchard Keeper, p. 8)

Dites donc, fit-il, vous auriez pas - et ses yeux dressèrent un rapide et dernier inventaire -, hum, des pompes pour gonfler les pneus, par hasard ? (Hirsch, F. \& P. Schaeffer, p. 12)

Now vise à instaurer un ajustement interénonciatif pour rendre possible l'échange dialogique. Halliday \& Hasan (1976 : 268) contrastent now déictique accentué à ce now cohésif non accentué qui apparaît tout particulièrement dans des situations de «transactions » : « it means the opening of a new stage in the communication; this may be a new incident in the story, a new point in the argument, a new role or attitude being taken on by the speaker, and so on. » Le rôle de now est de construire un domaine commun aux deux partenaires énonciatifs là où il n'y en avait pas. La construction de l'altérité se conçoit ici comme le passage d'une absence de relation intersubjective à un domaine commun permettant la coénonciation. La même opération sous-tend l'emploi de now à chaque fois que l'énonciateur rejette un état de choses:

Mick. I want a first-class experienced interior decorator. I thought you were one.

Davies. Me? Now wait a minute - wait a minute - you got the wrong man. (...)

Mick. I understood you were an experienced first-class professional interior and exterior decorator.

Davies. Now look here -

Mick. You mean you wouldn't know how to fit teal-blue, copper and parchment linoleum squares and have those colours re-echoed in the walls?

Davies. Now, look here, where'd you get -? (...)

Mick. You're a bloody impostor, mate!

Davies. Now you don't want to say that sort of thing to me. (H. Pinter, The Caretaker, p. 72)

Le locuteur est en désaccord avec le point de vue exprimé en p, et now indique qu'il cherche à améliorer cet état de choses en prenant en compte la différence de points de vue et en s'ajustant au point de vue de l'autre. Même si now apparaît de façon fréquente dans des dialogues, la situation d'interlocution n'est pas une condition nécessaire à son emploi. De la même façon, il peut structurer la relation au co-énonciateur dans toute argumentation (récit, essai, discours) comme en (8) ou comme dans l'exemple suivant :

$$
\begin{aligned}
& \text { I am here concerned more with policy than with men. Public men come and go but great issues } \\
& \text { of policy remain. Now, everyone knows- or knew in the week of December that something had } \\
& \text { gone shockingly wrong with American foreign policy. The United States was engaged in a } \\
& \text { military attack on a peaceful, orderly people governed by a regime that had proved itself the } \\
& \text { most pro-Western and anti-Communist within any of the new nations- the only place in Africa, } \\
& \text { moreover, where a productive relationship between whites and blacks had apparently been } \\
& \text { achieved. (Brown Corpus) }
\end{aligned}
$$

L'altérité introduite par now correspond à une prémisse et non au terme du raisonnement, ce qui le distingue de maintenant. On peut établir un parallèle entre now et or, qui était employé à la place de maintenant jusqu'au XVIème siècle. Or a perdu sa valeur 
temporelle et sa valeur déictique, exprimées par maintenant en français moderne, si bien que la référence à une simple altérité se fait avec or et non avec maintenant.

\subsubsection{Nun et la conformité}

Now et nun, en dépit de leur parenté, présentent des différences abordées en $1 . N u n^{7}$ ne peut être topic-changer ${ }^{8}$ comme now. L'altérité introduite est toujours conforme à p, ou en tout cas présentée comme telle ${ }^{9}$. On n'aurait donc pas nun dans des contextes comme ceux illustrés de (19) à (21). Si nun apparaît suivi d'une injonction, celle-ci renvoie non pas à un point de vue divergent mais à ce que l'énonciateur attend conformément à $\mathrm{p}$ :

$$
\begin{aligned}
& \text { Er zeigte mit dem Bambusstöckchen auf die Tafel. „Nun lies mal!“ (exemple emprunté à } \\
& \text { Metrich et al. p. 293) } \\
& \text { Il dirigea la pointe du bambou vers le tableau noir. } \\
& \text { - Eh bien, lis. }
\end{aligned}
$$

Etant donné que l'instituteur s'est aperçu au préalable que son fils savait lire, la suite est ici conforme à cette prise de conscience. Le rôle de nun est de manifester cette conformité au coénonciateur. Si nun apparaît seul, en mot phrase interrogatif selon la terminologie de Metrich $\&$ al., c'est également pour indiquer à l'interlocuteur qu'on attend qu'il agisse ou qu'il parle pour mettre l'état de choses en conformité avec la séquence précédente :

„Was ist ?“ fragte er. „Hast du mir noch etwas zu bestellen ? Nun ? Sag es nur !“ (P. Süskind, das Parfum, p. 92)

[Qu'y a-t-il ? demanda-t-il. Est-ce que tu as encore quelque chose à me dire ? Eh bien ? Parle !]

Ainsi, en (24), dans l'hypothèse où l'interlocuteur a effectivement encore quelque chose à dire, il serait logique qu'il s'exprime, et nun indique ici que l'absence de prise de parole n'est pas en adéquation avec cette hypothèse. Dans le cas de figure illustré par ces deux exemples, le locuteur justifie son injonction à l'interlocuteur en lui demandant de se mettre en conformité avec la séquence p. C'est parce que nun manifeste la conformité de q que son emploi peut s'accompagner de nuances d'impatience ou de désapprobation, comme dans l'expression nun, wird's bald? (alors, ça vient?), dans laquelle on fait apparaître que quelque chose d'attendu ne vient pas, pour mieux inciter l'interlocuteur à agir.

Inversement, le locuteur peut employer nun en interrogation pour indiquer à son interlocuteur qu'un état de choses lui paraît inattendu :

\footnotetext{
Als er ein Weilchen fortgegangen war, fand er einen Jagdhund auf dem Wege liegen. Der japte wie einer, der sich müde gelaufen hatte. „Nun? Was japst du so ?“, fragte der Esel? (Grimm, Die Bremerstadtmusikanten)

Comme il avait marché quelque temps, il rencontra sur le chemin un chien de chasse qui jappait comme un animal fatigué d'une longue course. «Qu'as-tu donc à japper de la sorte,
}

C'est également la différence la plus importante que Hasselgård met en évidence entre now et nå en norvégien : « The Norwegian continuative $n a ̊[. .$.$] seems to have an affective meaning (impatience, irritation, surprise) when it occurs on its own, typically as$ part of a response or as a marker of agreement in combination with vel or ja. » 
camarade? lui dit-il.» (Contes choisis des frères Grimm, traduits par F. Baudry, $<\mathrm{http}: / /$ www.fabellia.com/wmview.php?ArtID=217>)

En (25), où jappen « est le cas », l'interrogation avec nun met en question la conformité de jappen par rapport à l'état de choses présent. Il s'agit, comme le révèle ensuite la deuxième interrogation « was japst du so ? », de montrer à l'interlocuteur que jappen est contraire à la norme afin que ce dernier justifie ses actes ; l'interlocuteur peut également être amené à justifier son dire comme dans l'exemple suivant :

\author{
Frl Doktor: Inspektor. Fällt Ihnen nichts auf? \\ Inspektor: Inwiefern? \\ Frl Doktor: Denken Sie an die beiden Kranken. \\ Inspektor: Nun? \\ Frl Doktor: Beide sind Physiker, Kernphysiker. (F. Dürrenmatt, die Physiker) \\ [Mlle le docteur: Inspecteur. Il n'y a rien qui vous frappe? \\ Inspecteur : Dans quelle mesure? \\ Mlle le docteur : Pensez aux deux malades. \\ Inspecteur : Et alors? \\ Mlle le docteur : Ils sont tous deux physiciens, atomistes.]
}

C'est ici la conformité-même de p qui est mise en question par nun en interrogation. Du point de vue du locuteur, la séquence qui précède ne permet pas de continuer le raisonnement. La fonction de nun est ainsi largement orientée vers le coénonciateur au sens où la conformité du dire lui est constamment rappelée, comme s'il s'agissait de mettre à jour un consensus interénonciatif au fil du discours - ou de la narration.

En conclusion, maintenant et now sont susceptibles de fonctionner en tant qu'adverbes temporels ou marqueurs discursifs. En tant que marqueurs discursifs, ils marquent une altérité de point de vue, mais les points de référence ne sont pas les mêmes : le point de référence est l'énonciateur en français, ce qui donne lieu à une fonction réflexive, alors que le point de référence est le coénonciateur en anglais, ce qui permet de construire un domaine commun aux deux partenaires énonciatifs là où l'échange avait échoué, ou bien là où il n'y avait pas d'échange. Nun en allemand est un marqueur discursif dans tous ses emplois, y compris temporels. L'altérité qu'il introduit manifeste la conformité de la séquence introduite à la séquence précédente, ce qui permet de justifier le dire au co-énonciateur, ou, dans des contextes dialogiques, d'inciter l'interlocuteur à agir. Maintenant manifeste donc un point de vue discordant, tandis que now et nun construisent un ajustement à l'autre de deux façons différentes: en sollicitant directement son point de vue avec now, en convoquant la conformité du discours pour le justifier à l'autre avec nun.

\title{
Références bibliographiques
}

AiJMer, K., (2002), English Discourse Particles, Amsterdam, John Benjamins.

CELlE, A., (2003), « A contrastive approach to temporal deixis in English, French and German » dans COMRIE, B. \& SulHKonen, P. (éds.), International Symposium on Deictic Systems and Quantification in Languages Spoken in Europe and North and Central Asia. Udmurt State University, Izhevsk, Udmurt Republic, Russia, May 21-24, 2001, Collection of papers, Izhevsk \& Leipzig, Udmurt State University, \& Max Planck Institute for Evolutionary Anthropology, 17-31. 
CElle, A., (2004), «A propos des marqueurs discursifs now et maintenant» dans DelmAS, C. (éd.), La contradiction en anglais, CIEREC Travaux 116, Université de Saint-Etienne, 91-106.

CElle, A., (2005), Temps et modalité, l'anglais, le français et l'allemand en contraste, Berne, Peter Lang.

HALlidAY, M.A.K. \& HASAN, R., (1976), Cohesion in English, Londres, Longman.

HASSElgÅRD, H., (à paraître), " 'Not now' - on non-correspondence between the cognate adverbs now and nå”, dans Aijmer, K., Simon-VAndenbergen, A.-M. (éds), Pragmatic Markers in Contrast, Elsevier.

MEtrich, R., FAuChER, E. \& COURDIER, G., (2002), Les invariables difficiles, Dictionnaire allemand-français des particules, connecteurs, interjections et autres "mots de la communication", vol.II/3, CNRS / Université de Nancy 2, Bibliothèque des nouveaux cahiers d'allemand.

NYAN, T., (1998), Metalinguistic Operators with Reference to French, Berne, Peter Lang.

NEF, F. (1980), «Maintenant1 et maintenant 2 : sémantique et pragmatique de 'maintenant' temporel et non temporel » dans David, J. \& Martin, R. (éds), Recherches linguistiques V, La notion d'aspect, Paris, Librairie Klincksieck, 145-166.

PAILlARD, D., (à paraître), «Déjà: adverbe ou marqueur discursif? », communication au colloque Chronos 6 (Genève, septembre 2004).

SCHIFFrIN, D., (1987), Discourse Markers, Cambridge, Cambridge University Press. 